\title{
Group decision-making based on heterogeneous preference relations with self-confidence
}

\author{
Yucheng Dong, Wenqi Liu, \\ Business School, Sichuan University, Chengdu 610065, China \\ E-mail: ycdong@scu.edu.cn; wqliu@stu.scu.edu.cn \\ Francisco Chiclana, \\ Faculty of Technology, De Montfort University Leicester, United Kingdom \\ E-mail: chiclana@dmu.ac.uk \\ Enrique Herrera-Viedma \\ DECSAI, University of Granada, Granada, Spain \\ viedma@deecsai.ugr.es \\ Francisco Javier Cabrerizo \\ Dept. Software Engineering and Computer Systems, UNED \\ Madrid, Spain \\ cabrerizo@isis.uned.es
}

\begin{abstract}
Preference relations are very useful to express decision makers' preferences over alternatives in the process of group decision-making. However, the multiple self-confidence levels are not considered in existing preference relations. In this study, we define the preference relation with self-confidence by taking multiple self-confidence levels into consideration, and we call it the preference relation with self-confidence. Furthermore, we present a two-stage linear programming model for estimating the collective preference vector for the group decision-making based on heterogeneous preference relations with self-confidence. Finally, numerical examples are used to illustrate the two-stage linear programming model, and a comparative analysis is carried out to show how self-confidence levels influence on the group decision-making results.
\end{abstract}

Keywords: Preference relations, self-confidence levels, collective preference vector, linear programming model.

\section{Introduction}

Preference relations are widely used in group decision-making (GDM). An $n \times n$ complete preference relation contains $n^{2}$ preference elements, and each element indicates 
the degree up to which an alternative is preferred to another one [30, 31, 33-35]. Sometimes, decision makers have no self-confidence on the preference information because of time pressure and limited expertise regarding the problem domain. In these situations, decision makers may provide their preference information in the form of incomplete preference relations, i.e. a preference relation with some of its elements missing [1, 23, 24, 38-40].

In a complete preference relation, the decision maker provides all preference information, and it is generally assumed that all preference values are provided with the same self-confidence level. In an incomplete preference relation, two self-confidence levels are used: (1) The decision maker is of self-confidence for those preference elements for which a value is provided and (2) the decision maker is without self-confidence for the preference elements for which a value is not given.

However, multiple self-confidence levels different to the two levels case mentioned above are not considered in existing preference relations. Therefore, it would be of great importance to provide decision makers with tools to allow them to express multiple self-confidence levels when providing their preferences. In this study, we propose the preference relation with self-confidence by taking multiple self-confidence levels into consideration, and we call it the preference relation with self-confidence. In the preference relation with self-confidence, each element consists of two components, the first one is the preference value between pairs of alternatives, and the second part, which is defined on a linguistic terms set, represents the decision maker's self-confidence level of its corresponding first part or preference value.

In practical GDM problems, each decision maker has different knowledge, experience, culture and educational backgrounds. As a result, the decision makers use different preference relations to express their individual preference information. Three kinds of preference relations have been widely investigated: multiplicative preference relations [5, 26, 33, 41], additive preference relations $[6,19,31,34-36,41]$ and linguistic preference relations $[8,9$, 11, 16, 20, 28, 37]. Chiclana et al. [4, 5], Dong et al. [12, 14], Herrera et al. [26], and Herrera-Viedma et al. [21] initiated and developed the GDM models with heterogeneous preference relations represented by preference orderings, utility functions, additive preference relations, multiplicative preference relations. Moreover, Fan et al. [18] and Ma et al. [29] initiated several optimization-based models to integrate heterogeneous preference relations. The GDM problem with heterogeneous preference relations has become one of the major areas of GDM researches [3]. 
An important challenge to bear in mind when decision makers provide different preference relations with self-confidence is how to obtain the collective solution. In this paper, a two-stage linear programming model to deal with GDM problems based on heterogeneous preference relations with self-confidence is developed. This two-stage linear programming model is based on a distance-based framework that minimizes the information deviation between decision makers' preference relations and collective preference vector, and that is presented first. .

The rest of this study is organized as follows. Section 2 introduces the basic knowledge regarding ordinal linguistic 2-tuples model and the three kinds of preference relations mentioned above. Section 3 defines the preference relations with self-confidence and describes the GDM problem based on heterogeneous preference relations with self-confidence. Section 4 proposes a distance-based framework that is used to develop a two-stage linear programming model for estimating the collective preference vector in the GDM. Section 5 provides numerical examples and a comparative analysis to show how self-confidence levels influence on the GDM results. Finally, Section 6 concludes the study.

\section{Preliminaries}

In this section, and with the aim of making this study self-contained, preliminary concepts regarding the ordinal linguistic 2-tuple model and the three main type of preference relations used in this framework are covered.

\subsection{The ordinal 2-tuple linguistic model}

The ordinal 2-tuple linguistic model is used in this study to carry out ordinal computing with words when dealing with the linguistic self-confidence levels information. The basic notations and operational laws of ordinal linguistic variables are introduced in [13, 15, 25, 27], a summary of which is provided below.

Let $S=\left\{s_{i} \mid i=0,1, \ldots, g\right\}$ be a linguistic term set with odd cardinality. The term $s_{i}$ represents a possible value of a linguistic variable. The following ordinal ordering on set $S$ is assumed:

$$
s_{i}>s_{j} \text { if and only if } i>j
$$

Herrera and Martínez presented the ordinal 2-tuple linguistic model in [25], and it was based in the following adapted definition: 
Definition 1 (Herrera and Martínez [25]): Let $\beta \in[0, g]$ be a number in the granularity interval of the linguistic term set $S=\left\{s_{0}, \ldots, s_{g}\right\}$ and let $i=\operatorname{round}(\beta)$ and $\alpha=\beta-i$ be two values such that $i \in[0, g]$ and $\alpha \in[-0.5,0.5)$. Then, $\alpha$ is called a symbolic translation, with round being the usual rounding operation.

Herrera and Martínez's model represents the linguistic information by means of ordinal 2-tuples $\left(s_{i}, \alpha\right)$, where $s_{i}$ is a simple term in $S$ and $\alpha \in[-0.5,0.5)$. A one-to-one mapping between ordinal linguistic 2 -tuples and numerical values in $[0, \mathrm{~g}]$ is possible.

Definition 2 (Herrera and Martínez [25]): Let $S=\left\{s_{0}, \ldots, s_{g}\right\}$ be a linguistic term set and $\beta \in[0, g]$ a value representing the result of a symbolic aggregation operation, then the ordinal 2-tuple that expresses the equivalent information to $\beta$ is obtained with the following function: $\Delta:[0, g] \rightarrow S \times[-0.5,0.5)$, where

$$
\Delta(\beta)=\left(s_{i}, \alpha\right), \text { with }\left\{\begin{array}{l}
s_{i}, i=\operatorname{round}(\beta) \\
\alpha=\beta-i, \alpha \in[-0.5,0.5) .
\end{array}\right.
$$

For convenience, denoting by $\bar{S}=S \times[-0.5,0.5)$ the inverse function of $\Delta$ is $\Delta^{-1}: \bar{S} \rightarrow[0, g]$ with $\Delta^{-1}\left(\left(s_{i}, \alpha\right)\right)=i+\alpha$. For notation simplicity, this paper sets $\Delta^{-1}\left(\left(s_{i}, 0\right)\right)=\Delta^{-1}\left(s_{i}\right)$. Clearly, an ordering on the set of ordinal 2-tuples and a negation operator are possible to define as follows:

1) Let $\left(s_{k}, \alpha\right)$ and $\left(s_{l}, \gamma\right)$ be two ordinal 2-tuples. Then:

(1) if $k<l$, then $\left(s_{k}, \alpha\right)$ is smaller than $\left(s_{l}, \gamma\right)$.

(2) if $k=l$, then

(a) if $\alpha=\gamma$, then $\left(s_{k}, \alpha\right)$ and $\left(s_{l}, \gamma\right)$ represents the same information.

(b) if $\alpha<\gamma$, then $\left(s_{k}, \alpha\right)$ is smaller than $\left(s_{l}, \gamma\right)$.

2) Ordinal 2-tuple negation operator:

$$
\operatorname{Neg}\left(\left(s_{i}, \alpha\right)\right)=\Delta\left(g-\left(\Delta^{-1}\left(s_{i}, \alpha\right)\right)\right)
$$

\subsection{Preference relations}

In this subsection, we introduce multiplicative preference relations, additive preference relations and ordinal 2-tuple linguistic preference relations.

(1) Multiplicative preference relations

Saaty introduced multiplicative preference relations in [33].

Definition 3 [33]: Let $X=\left\{x_{1}, x_{2}, \ldots, x_{n}\right\}$ be a finite set of alternatives. A multiplicative preference relation $A=\left(a_{i j}\right)_{n \times n}$ on $X$ is described by a positive preference relation 
$A \subset X \times X$, with element $a_{i j}$ measuring on a ratio scale $[1 / 9,9]$ the intensity of preference of alternative $x_{i}$ over alternative $x_{j}$. The following interpretation is assumed: $a_{i j}=1$ indicates indifference between $x_{i}$ and $x_{j} ; a_{i j}=9$ indicates that $x_{i}$ is absolutely preferred to $x_{j}$, and $a_{i j} \in\{2,3, \ldots, 8\}$ indicates intermediate evaluations. It is assumed that $a_{i j} a_{j i}=1$ and $a_{i i}=1$.

(2) Additive preference relations

Additive preference relations are also called fuzzy preference relations [4, 21, 31].

Definition 4 [31]: An additive preference relation $P$ on a finite set of alternatives $X$ is a relation in $X \times X$ that is characterised by a membership function $\mu_{P}: X \times X \rightarrow[0,1]$, where $\mu_{P}\left(x_{i}, x_{j}\right)=p_{i j}$ denotes the preference degree or intensity of the alternative $x_{i}$ over $x_{j}$. The following interpretation is assumed: $p_{i j}=0.5$ indicates indifference between $x_{i}$ and $x_{j}, p_{i j}>0.5$ indicates a definite preference for $x_{i}$ over $x_{j}$, $p_{i j}=1$ indicates the maximum degree of preference for $x_{i}$ over $x_{j}$. It is assumed that $p_{i j}+p_{j i}=1$ and $p_{i i}=0.5$.

(3) Ordinal 2-tuple linguistic preference relations

Let $S=\left\{s_{i} \mid i=0,1, \ldots, g\right\}$ be an ordinal linguistic term set with odd cardinality as introduced in Section 2.1.

Definition 5 [27]: An ordinal 2-tuple linguistic preference relation $T$ on a finite set of alternatives $X$ is defined as $T=\left(t_{i j}\right)_{n \times n}$, where $t_{i j} \in S$ denotes the degree of linguistic preference of the alternative $x_{i}$ over $x_{j}$. The following interpretation is assumed: $t_{i j}=s_{\frac{g}{2}}$ indicates indifference between $x_{i}$ and $x_{j}, t_{i j}>s_{\frac{g}{2}}$ indicates a definite preference for $x_{i}$ over $x_{j}$, and $t_{i j}<s_{\frac{g}{2}}$ indicates a definite preference for $x_{j}$ over $x_{i}$. It is assumed that $t_{i i}=s_{\frac{g}{2}}$ and $t_{i j}=\operatorname{Neg}\left(t_{j i}\right)$. 
Initially, the decision maker expresses her/his preferences using the simple ordinal terms of $S$, and the ordinal 2-tuple linguistic values only appear after operations on simple ordinal terms are carried out.

\subsection{Transitivity of preferences}

Transitivity is an important concept to apply to preference relations to assess their rationality. Here, we list there different transitive properties of preference relations, with the third one being a stronger condition than the second one, which in turn is stronger than the first one.

Let $A=\left(a_{i j}\right)_{n \times n}$ be a multiplicative preference relation. Some transitive properties of multiplicative preference relations can be described as follows:

(a) Weak stochastic transitivity: $a_{i j} \geq 1, a_{j k} \geq 1 \Rightarrow a_{i k} \geq 1 \forall i, j, k$.

(b) Strong stochastic transitivity: $a_{i j} \geq 1, a_{j k} \geq 1 \Rightarrow a_{i k} \geq \max \left(a_{i j}, a_{j k}\right) \forall i, j, k$.

(c) Multiplicative transitivity: $a_{i j} a_{j k}=a_{i k} \forall i, j, k$.

The equivalent properties for additive preference relations and 2-tuple linguistic preference relations are also described as follows. Let $P=\left(p_{i j}\right)_{n \times n}$ be an additive preference relation.

(a) Weak stochastic transitivity: $p_{i j} \geq 0.5, p_{j k} \geq 0.5 \Rightarrow p_{i k} \geq 0.5 \forall i, j, k$.

(b) Strong stochastic transitivity: $p_{i j} \geq 0.5, p_{j k} \geq 0.5 \Rightarrow p_{i k} \geq \max \left(p_{i j}, p_{j k}\right) \forall i, j, k$.

(c) Additive transitivity: $p_{i j}=p_{i k}-p_{j k}+0.5 \forall i, j, k$.

Let $T=\left(t_{i j}\right)_{n \times n}$ be an ordinal 2-tuple linguistic preference relation.

(a) Weak stochastic transitivity: $t_{i j} \geq s_{\frac{g}{2}}, t_{j k} \geq s_{\frac{g}{2}} \Rightarrow t_{i k} \geq s_{\frac{g}{2}} \forall i, j, k$.

(b) Strong stochastic transitivity: $t_{i j} \geq s_{\frac{g}{2}}, t_{j k} \geq s_{\frac{g}{2}} \Rightarrow t_{i k} \geq \max \left(t_{i j}, t_{j k}\right) \forall i, j, k$.

(c) Additive transitivity: $\Delta^{-1}\left(t_{i j}\right)=\Delta^{-1}\left(t_{i k}\right)-\Delta^{-1}\left(t_{j k}\right)+\frac{g}{2} \forall i, j, k$.

A preference relation that verifies the stronger of the above three transitivity properties is usually referred to as a consistent preference relation following Saaty's definition of consistency of multiplicative preference relations. 


\section{Preference relations with self-confidence in GDM}

In this section, we define three kinds of preference relations with self-confidence and describe the GDM problem based on heterogeneous preference relations with self-confidence.

To enable decision makers to characterize self-confidence levels in a linguistic way, a linguistic terms set $S^{S L}=\left\{l_{0}, l_{1}, \ldots, l_{g}\right\}$ is used, with the following one being a possible example:

$$
\begin{gathered}
S^{S L}=\left\{l_{0}=\text { extremely poor, } l_{1}=\text { very poor }, l_{2}=\text { poor }, l_{3}=\text { slightly poor, } l_{4}=\text { fair },\right. \\
\left.l_{5}=\text { slightly good, } l_{6}=\text { good }, l_{7}=\text { very good }, l_{8}=\text { extremely good }\right\}
\end{gathered}
$$

The decision maker uses the simple term $l_{i} \in S^{S L}$ to characterize his/her self-confidence level over the preference value.

\subsection{Preference relations with self-confidence}

Definitions of multiplicative preference relation with self-confidence, additive preference relation with self-confidence and ordinal 2-tuple linguistic preference relation with self-confidence on a finite set of alternatives $X=\left\{x_{1}, x_{2}, \ldots, x_{n}\right\}$ are given below:

Definition 6: A multiplicative preference relation with self-confidence on a finite set of alternatives $X, A^{*}=\left(\left(a_{i j}, s_{i j}\right)\right)_{n \times n}$, is relation on $X \times X$ whose elements have two components, the first one $a_{i j} \in[1 / 9,9]$ representing the preference degree or intensity of the alternative $x_{i}$ over $x_{j}$, and the second component $s_{i j} \in S^{S L}$ representing the self-confidence level associated to the first component. The following conditions are assumed: $a_{i j} a_{j i}=1, a_{i i}=1, s_{i j}=s_{j i}$ and $s_{i i}=l_{g}$.

Definition 7: An additive preference relation with self-confidence on a finite set of alternatives $X, P^{*}=\left(\left(p_{i j}, s_{i j}\right)\right)_{n \times n}$, is relation on $X \times X$ whose elements have two components, the first one $p_{i j} \in[0,1]$ representing the preference degree or intensity of the alternative $x_{i}$ over $x_{j}$, and the second component $s_{i j} \in S^{S L}$ representing the self-confidence level associated to the first component. The following conditions are assumed: $p_{i j}+p_{j i}=1, \quad p_{i i}=0.5, s_{i j}=s_{j i}$ and $s_{i i}=l_{g}$. 
Definition 8: An ordinal 2-tuple linguistic preference relation with self-confidence on a finite set of alternatives $X, T^{*}=\left(\left(t_{i j}, s_{i j}\right)\right)$, is relation on $X \times X$ whose elements have two components, the first one $t_{i j} \in S$ representing the ordinal 2-tuple linguistic preference of the alternative $x_{i}$ over $x_{j}$, and the second component $s_{i j} \in S^{S L}$ representing the self-confidence level associated to the first component. The following conditions are assumed: $t_{i j}=\operatorname{Neg}\left(t_{j i}\right), t_{i i}=s_{\frac{g}{2}}, s_{i j}=s_{j i}$ and $s_{i i}=l_{g}$.

Remark: Zadeh [42] developed the concept of a Z-number relates to the issue of reliability of information. A Z-number is an ordered pair of fuzzy numbers, the first component is a restriction (constraint) on the values which a real-valued uncertain variable, and the second component is a measure of reliability (certainty) of the first component. In our preference relation with self-confidence, each element can be considered to be a Z-number (in some sense).

In the following, we describe some transitive properties of preference relations with self-confidence.

Let $A^{*}=\left(\left(a_{i j}, s_{i j}\right)\right)_{n \times n}$ be a multiplicative preference relation with self-confidence. Some transitive properties can be described as follows:

(a) Weak stochastic transitivity at the self-confidence level $l \in S^{S L}$.

$$
a_{i j} \geq 1, a_{j k} \geq 1 \Rightarrow a_{i k} \geq 1, \forall i, j, k \text { and } s_{i j} \geq l, \forall i, j .
$$

(b) Strong stochastic transitivity at the self-confidence level $l \in S^{S L}$.

$$
a_{i j} \geq 1, a_{j k} \geq 1 \Rightarrow a_{i k} \geq \max \left(a_{i j}, a_{j k}\right), \forall i, j, k \text { and } s_{i j} \geq l, \forall i, j .
$$

(c) Multiplicative transitivity at the self-confidence level $l \in S^{S L}$.

$$
a_{i j} a_{j k}=a_{i k}, \forall i, j, k \text { and } s_{i j} \geq l, \forall i, j .
$$

Let $P^{*}=\left(\left(p_{i j}, s_{i j}\right)\right)_{n \times n}$ be an additive preference relation with self-confidence.

(a) Weak stochastic transitivity at the self-confidence level $l \in S^{S L}$.

$$
p_{i j} \geq 0.5, p_{j k} \geq 0.5 \Rightarrow p_{i k} \geq 0.5, \forall i, j, k \text { and } s_{i j} \geq l, \forall i, j .
$$

(b) Strong stochastic transitivity at the self-confidence level $l \in S^{S L}$.

$$
p_{i j} \geq 0.5, p_{j k} \geq 0.5 \Rightarrow p_{i k} \geq \max \left(p_{i j}, p_{j k}\right), \forall i, j, k \text { and } s_{i j} \geq l, \forall i, j .
$$

(c) Additive transitivity at the self-confidence level $l \in S^{S L}$. 


$$
p_{i j}=p_{i k}-p_{j k}+0.5, \forall i, j, k \text { and } s_{i j} \geq l, \forall i, j
$$

Let $T^{*}=\left(\left(t_{i j}, s_{i j}\right)\right)_{n \times n}$ be an ordinal 2-tuple linguistic preference relation with self-confidence.

(a) Weak stochastic transitivity at the self-confidence level $l \in S^{S L}$.

$$
t_{i j} \geq s_{\frac{g}{2}}, t_{j k} \geq s_{\frac{g}{2}} \Rightarrow t_{i k} \geq s_{\frac{g}{2}}, \forall i, j, k \text { and } s_{i j} \geq l, \forall i, j .
$$

(b) Strong stochastic transitivity at the self-confidence level $l \in S^{S L}$.

$$
t_{i j} \geq s_{\frac{g}{2}}, t_{j k} \geq s_{\frac{g}{2}} \Rightarrow t_{i k} \geq \max \left(t_{i j}, t_{j k}\right), \forall i, j, k \text { and } s_{i j} \geq l, \forall i, j .
$$

(c) Additive transitivity at the self-confidence level $l \in S^{S L}$.

$$
\Delta^{-1}\left(t_{i j}\right)=\Delta^{-1}\left(t_{i k}\right)-\Delta^{-1}\left(t_{j k}\right)+\frac{g}{2}, \forall i, j, k \text { and } s_{i j} \geq l, \forall i, j .
$$

The traditional definition to characterize consistency of preference relations is using a set of pre-established transitive properties $[1,6,22]$. In this paper, a preference relation with self-confidence is considered to be acceptable consistent if it satisfies the weak stochastic transitivity at the self-confidence level $l_{0} \in S^{S L}$.

\subsection{Group decision-making problem with self-confidence}

Let $X=\left\{x_{1}, x_{2}, \ldots, x_{n}\right\}$ be a finite set of $n$ alternatives. These alternatives have to be classified from best to worst, using the information given by a finite set of decision makers $E=\left\{e_{1}, e_{2}, \ldots, e_{m}\right\}$. Let $C=\left\{c_{1}, c_{2}, \ldots, c_{m}\right\}$ be a set of normalized weight/importance values associated to the set of experts: $c_{k}$ is the weight/importance value of decision maker

$e_{k}$ and $c_{k} \geq 0, \quad \sum_{k=1}^{m} c_{k}=1$. As each decision maker $e_{k} \in E$ has their own ideas, attitudes, motivations, and personality, it is quite natural to consider that different decision makers will give their preferences in a different way. Thus, decision makers' preferences over the set of alternatives $X$ may be represented in one of the following three ways: multiplicative preference relations with self-confidence, additive preference relations with self-confidence and ordinal 2-tuple linguistic preference relations with self-confidence. Without loss of generality, let $E^{A^{*}}=\left\{e_{1}, e_{2}, \ldots, e_{m_{1}}\right\}, E^{P^{*}}=\left\{e_{m_{1}+1}, e_{m_{1}+2}, \ldots, e_{m_{2}}\right\}, E^{T^{*}}=\left\{e_{m_{2}+1}, e_{m_{2}+2}, \ldots, e_{m}\right\}$ be three subsets of $E$, representing the set of decision makers whose preference information on $X$ are expressed as multiplicative preference relations with self-confidence, additive preference relations with self-confidence and ordinal 2-tuple linguistic preference relations 
with self-confidence, respectively. The question is how to obtain a collective solution to the GDM problem based on heterogeneous preference relations with self-confidence level.

\section{A two-stage linear programming model}

In this section, we first propose a distance-based framework that aims to minimize the information deviation between decision makers' preference relations and the collective preference vector, which later is used to develop a two-stage linear programming model to solve the GDM problem at hand.

\subsection{A distance-based framework}

Let $w^{c}=\left(w_{1}^{c}, w_{2}^{c}, \ldots, w_{n}^{c}\right)^{T}$ be the collective priority preference vector of the decision makers, where $\sum_{i=1}^{n} w_{i}^{c}=1$ and $w_{i}^{c} \geq 0$ for $\forall i$. In general, there are differences between the individual preference information and the collective solution, which can be measured as follows:

(1) Let $A^{* k}=\left(\left(a_{i j}^{k}, s_{i j}^{k}\right)\right)_{n \times n}$ be a multiplicative preference relation with self-confidence. The error between the preference value $a_{i j}^{k}$ and the collective priority preference vector $w^{c}$ is $[18,33]$

$$
\varepsilon_{i j}^{k}=w_{i}^{c}-w_{j}^{c} a_{i j}^{k}, \quad k=1,2, \ldots, m_{1}, \quad i, j=1,2, \ldots, n
$$

(2) Let $P^{* k}=\left(\left(p_{i j}^{k}, s_{i j}^{k}\right)\right)_{n \times n}$ be an additive preference relation with self-confidence. The error between the preference value $p_{i j}^{k}$ and the collective priority preference vector $w^{c}$ is $[18,31]$

$$
\varepsilon_{i j}^{k}=\frac{1}{2}\left(w_{i}^{c}-w_{j}^{c}\right)+0.5-p_{i j}^{k}, \quad k=m_{1}+1, m_{1}+2, \ldots, m_{2}, \quad i, j=1,2, \ldots, n
$$

(3) Let $T^{* k}=\left(\left(t_{i j}^{k}, s_{i j}^{k}\right)\right)_{n \times n}$ be an ordinal 2-tuple linguistic preference relation with self-confidence. The error between the preference value $t_{i j}^{k}$ and the collective priority preference vector $w^{c}$ can be similarly defined as

$$
\varepsilon_{i j}^{k}=\frac{1}{2}\left(w_{i}^{c}-w_{j}^{c}\right)+\frac{g}{2}-\Delta^{-1}\left(t_{i j}^{k}\right), \quad k=m_{2}+1, m_{2}+2, \ldots, m, i, j=1,2, \ldots, n
$$

If the individual preference relations are consistent, then it is $\varepsilon_{i j}^{k}=0$. 
When the error $\varepsilon_{i j}^{k}$ is at a self-confidence level of $s_{i j}^{k}\left(s_{i j}^{k} \in S^{S L}\right)$, the following information deviation can be introduced

$$
z_{i j}^{k}=\left|\Delta^{-1}\left(s_{i j}^{k}\right)\right|\left|\varepsilon_{i j}^{k}\right|, \quad k=1,2, \ldots, m, \quad i, j=1,2, \ldots, n
$$

The level of self-confidence $s_{i j}^{k}$ in Eq. (8) determines the magnification of error $\varepsilon_{i j}^{k}$ : the larger its value, the larger magnification will be the error $\varepsilon_{i j}^{k}$ assigned to the corresponding preference value.

In the following, a distance-based framework that minimizes the information deviation between decision makers' preference relations and the collective preference vector is introduced. The following objective function are introduced for metric $1 \leq p \leq \infty$,

$$
\min \mathrm{z}=\sum_{k=1}^{m} c_{k} \alpha_{k}\left(\sum_{i=1}^{n} \sum_{j=1}^{n}\left(z_{i j}^{k}\right)^{p}\right)^{1 / p}
$$

where $\alpha_{k}$ is a normalization coefficient. Due to the varying domains adopted for the variety of preference formats, $\alpha_{k}$ is used to normalize the measure of the information deviation of each decision maker to eliminate the influence of heterogeneous preference relations in GDM. The normalization coefficients $\alpha_{k}$ is determined based on the size of the coefficient matrix $G^{k}$. Generally, according to matrix theory and related research in [29], the value of $\alpha_{k}$ can be calculated as follows,

$$
\alpha_{k}=\frac{1}{s p_{k}}, \quad k=1,2, \ldots, m
$$

where $s p_{k}$ is the Frobenius norm of matrix $G^{k}$. When $G^{k}$ has real number elements, the Frobenius norm is $s p_{k}=\sqrt{\lambda_{m}}$, where $\lambda_{m}$ is the greatest eigenvalue of $\left(G^{k}\right)^{T} G^{k}$.

The objective function (9) is affected by parameter $p$ : the 1-norm distance $(p=1)$ is Manhattan distance; the 2-norm distance $(p=2)$ is the Euclidean distance; the infinity norm distance $(p=\infty)$ is the Chebyshev distance. In this study, we study the 1-norm and the infinity norm distances. For $p=1$ and $p=\infty$, the above objective functions are expressed as Eq. (11) and (12), respectively.

$$
\min \mathrm{z}_{1}=\sum_{k=1}^{m} c_{k} \alpha_{k} \sum_{i=1}^{n} \sum_{j=1}^{n} z_{i j}^{k}
$$




$$
\min \mathrm{z}_{2}=\sum_{k=1}^{m} c_{k} \alpha_{k}\left(\max _{i, j=1,2, \ldots, n} z_{i j}^{k}\right)
$$

\subsection{A two-stage linear programming model based on the distance-based framework}

In this subsection, we develop a two-stage linear programming model for estimating the collective preference vector for the GDM, which is based on the previously introduced distance-based framework. In the first stage, we set $p=1$ to minimize the sum of all information deviation of all decision makers to obtain a set of collective preference vectors. In the second stage, we set $p=\infty$ and minimize the maximal information deviation of decision makers to select the optimal collective preference vector from the solution set of the first stage.

(1) First stage: $p=1$

We use three transformed variables in model (11): $y_{i j}=\varepsilon_{i j}, d_{i j}^{k}=\Delta^{-1}\left(t_{i j}^{k}\right)$ and $b_{i j}^{k}=\Delta^{-1}\left(s_{i j}^{k}\right)$. The first stage linear programming model is expressed as follows:

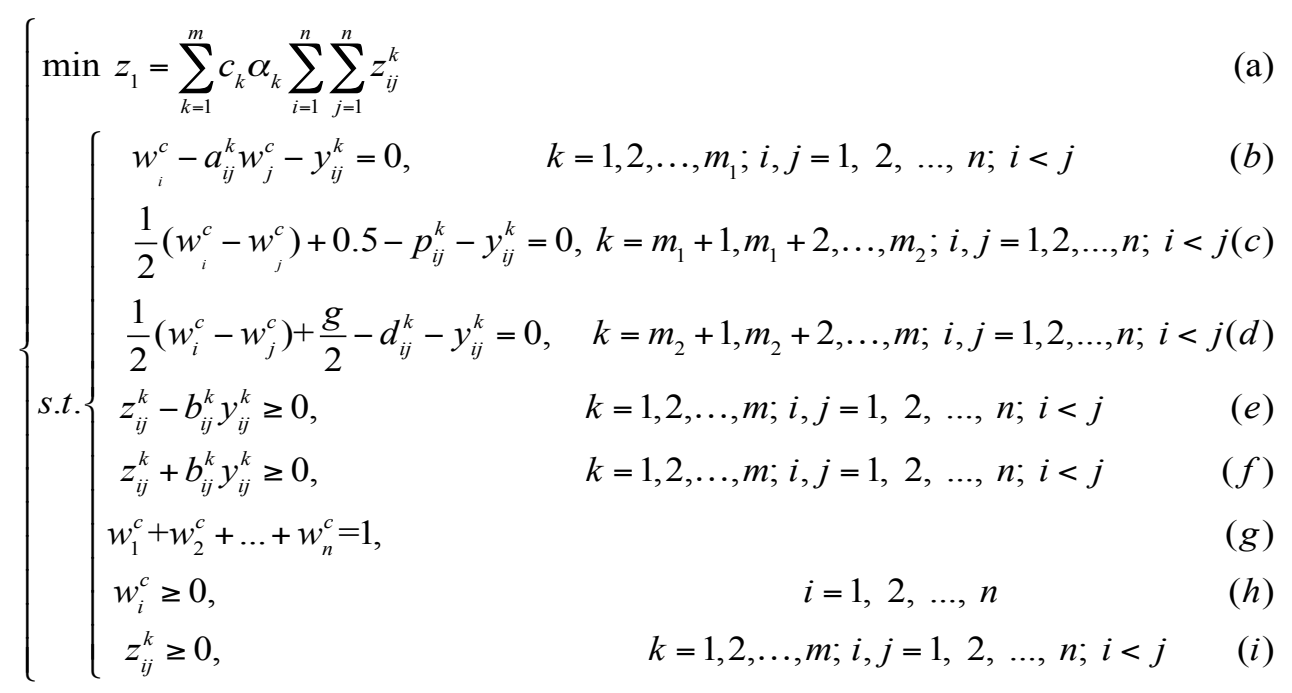

In model (13), constraints $(b)-(d)$ express the errors between the individual preference information and the collective preference vector; constraints $(e)$ and $(f)$ guarantee that $z_{i j}^{k} \geq\left|\Delta^{-1}\left(s_{i j}^{k}\right)\right|\left|\varepsilon_{i j}^{k}\right|$; constraint $(g)$ guarantees that the priority vector is normalized to sum to one; and finally, constraints $(h)$ and $(i)$ guarantee that variables $w_{i}^{c}$ and $z_{i j}^{c}$ are nonnegative.

(2) Second stage: $p=\infty$

It is possible that there are multiple optimal solutions to the first stage model. The second stage model is model (12), and further selects the optimal collective preference vector 
from the optimal solutions of the first stage model. The second stage linear programming model is given as follows:

$$
\left\{\begin{array}{l}
\min z_{2}=\sum_{k=1}^{m} c_{k} \alpha_{k}\left(z_{\max }^{k}\right) \\
s . t . \\
\begin{array}{ll}
\sum_{k=1}^{m} c_{k} \alpha_{k} \sum_{i=1}^{n} \sum_{j=1}^{n} z_{i j}^{k}=z_{1} \\
w_{i}^{c}-a_{i j}^{k} w_{j}^{c}-y_{i j}^{k}=0, \\
\frac{1}{2}\left(w_{i}^{c}-w_{j}^{c}\right)+0.5-p_{i j}^{k}-y_{i j}^{k}=0, & k=m_{1}+1, m_{1}+2, \ldots, m_{2} ; i, j=1,2, \ldots, n ; i<j(d) \\
\frac{1}{2}\left(w_{i}^{c}-w_{j}^{c}\right)+\frac{g}{2}-d_{i j}^{k}-y_{i j}^{k}=0, & k=m_{2}+1, m_{2}+2, \ldots, m ; i, j=1,2, \ldots, n ; i<j(e) \\
z_{i j}^{k}-b_{i j}^{k} y_{i j}^{k} \geq 0, & k=1,2, \ldots, m ; i, j=1,2, \ldots, n ; i<j \\
z_{i j}^{k}+b_{i j}^{k} y_{i j}^{k} \geq 0, & k=1,2, \ldots, m ; i, j=1,2, \ldots, n ; i<j \\
z_{\max }^{k}-z_{i j}^{k} \geq 0, & k=1,2, \ldots, m ; i, j=1,2, \ldots, n ; i<j \\
w_{1}^{c}+w_{2}^{c}+\ldots+w_{n}^{c}=1, & i=1,2, \ldots, n \\
w_{i}^{c} \geq 0, & k=1,2, \ldots, m ; i, j=1,2, \ldots, n ; i<j \\
z_{i j}^{k} \geq 0, & (f)
\end{array}
\end{array}\right.
$$

In model (14), constraint $(b)$ ensures that only those optimal solution(s) to the first stage model are feasible in the second stage model, and constraint $(h)$ finds $z_{\max }^{k}$. The rest of constraints are identical to the constraints of the first stage model. The two-stage linear programming model is straightforward and easy to understand and formulate, and it can be solved in very little computational time using readily available software such as LINGO.

\section{Numerical analysis}

In this section, we use three numerical examples to illustrate our two-stage linear programming model, and then we make a comparative analysis to show the influence of self-confidence levels on the group decision making results.

\subsection{Example 1}

We consider the following example, which includes three decision makers $e_{k}(k=1,2,3)$ and four alternatives $x_{i}(i=1,2,3,4)$. Suppose that the importance degree of each decision maker is equal, $c_{k}=1 / 3, k=1,2,3$. The decision maker $e_{1}$ provides his/her preference information by the multiplicative preference relation with self-confidence $A^{* 1}$, the decision maker $e_{2}$ provides his/her preference information by the additive preference relation with self-confidence $P^{* 2}$, the decision maker $e_{3}$ provides his/her preference information by the ordinal 2-tuple linguistic preference relation with self-confidence $T^{* 3}$. These preference relations with self-confidence satisfy the weak stochastic transitivity at the self-confidence level $l_{0}$. 


$$
\begin{aligned}
A^{* 1} & =\left(\begin{array}{llll}
\left(1, l_{8}\right) & \left(2, l_{5}\right) & \left(\frac{1}{3}, l_{6}\right) & \left(4, l_{3}\right) \\
\left(\frac{1}{2}, l_{5}\right) & \left(1, l_{8}\right) & \left(\frac{1}{3}, l_{4}\right) & \left(\frac{1}{4}, l_{2}\right) \\
\left(3, l_{6}\right) & \left(3, l_{4}\right) & \left(1, l_{8}\right) & \left(7, l_{6}\right) \\
\left(\frac{1}{4}, l_{3}\right) & \left(4, l_{2}\right) & \left(\frac{1}{7}, l_{6}\right) & \left(1, l_{8}\right)
\end{array}\right) \\
P^{* 2} & =\left(\begin{array}{llll}
\left(0.5, l_{8}\right) & \left(0.5, l_{5}\right) & \left(0.6, l_{2}\right) & \left(0.9, l_{3}\right) \\
\left(0.5, l_{5}\right) & \left(0.5, l_{8}\right) & \left(0.8, l_{6}\right) & \left(0.6, l_{4}\right) \\
\left(0.4, l_{2}\right) & \left(0.2, l_{6}\right) & \left(0.5, l_{8}\right) & \left(0.8, l_{7}\right) \\
\left(0.1, l_{3}\right) & \left(0.4, l_{4}\right) & \left(0.2, l_{7}\right) & \left(0.5, l_{8}\right)
\end{array}\right) \\
T^{* 3} & =\left(\begin{array}{llll}
\left(s_{4}, l_{8}\right) & \left(s_{1}, l_{5}\right) & \left(s_{1}, l_{2}\right) & \left(s_{4}, l_{4}\right) \\
\left(s_{7}, l_{5}\right) & \left(s_{4}, l_{8}\right) & \left(s_{6}, l_{3}\right) & \left(s_{4}, l_{7}\right) \\
\left(s_{7}, l_{2}\right) & \left(s_{2}, l_{3}\right) & \left(s_{4}, l_{8}\right) & \left(s_{3}, l_{0}\right) \\
\left(s_{4}, l_{4}\right) & \left(s_{4}, l_{7}\right) & \left(s_{5}, l_{0}\right) & \left(s_{4}, l_{8}\right)
\end{array}\right)
\end{aligned}
$$

Based on the first stage model (13), we can obtain the value of objective function $z_{1}=2.72$. Based on the second stage model (14), we can obtain $z_{2}=0.71$ and the collective preference vector $w^{c}=(0.26,0.26,0.42,0.06)^{T}$.

\subsection{Example 2}

We consider the second example, which includes four decision makers $e_{k}(k=1,2,3,4)$ and three alternatives $x_{i}(i=1,2,3)$. Suppose that the importance degree of each decision maker is equal, $c_{k}=1 / 4, k=1,2,3,4$. The decision maker $e_{1}$ provides his/her preference information by the multiplicative preference relation with self-confidence $A^{* 1}$, the decision makers $e_{2}$ and $e_{3}$ provide their preference information by the additive preference relations with self-confidence $P^{* 2}$ and $P^{* 3}$, the decision maker $e_{4}$ provides his/her preference information by the ordinal 2-tuple linguistic preference relation with self-confidence $T^{* 4}$. The corresponding matrices are given as follows, 


$$
\begin{aligned}
A^{* 1} & =\left(\begin{array}{lll}
\left(1, l_{8}\right) & \left(2, l_{6}\right) & \left(3, l_{7}\right) \\
\left(\frac{1}{2}, l_{6}\right) & \left(1, l_{8}\right) & \left(\frac{1}{4}, l_{5}\right) \\
\left(\frac{1}{3}, l_{7}\right) & \left(4, l_{5}\right) & \left(1, l_{8}\right)
\end{array}\right) \\
P^{* 2} & =\left(\begin{array}{lll}
\left(0.5, l_{8}\right) & \left(0.3, l_{5}\right) & \left(0.4, l_{4}\right) \\
\left(0.7, l_{5}\right) & \left(0.5, l_{8}\right) & \left(0.2, l_{2}\right) \\
\left(0.6, l_{4}\right) & \left(0.8, l_{2}\right) & \left(0.5, l_{8}\right)
\end{array}\right) \\
P^{* 3} & =\left(\begin{array}{lll}
\left(0.5, l_{8}\right) & \left(0.4, l_{6}\right) & \left(0.9, l_{5}\right) \\
\left(0.6, l_{6}\right) & \left(0.5, l_{8}\right) & \left(0.5, l_{0}\right) \\
\left(0.1, l_{5}\right) & \left(0.5, l_{0}\right) & \left(0.5, l_{8}\right)
\end{array}\right) \\
T^{* 4} & =\left(\begin{array}{lll}
\left(s_{4}, l_{8}\right) & \left(s_{3}, l_{6}\right) & \left(s_{8}, l_{4}\right) \\
\left(s_{5}, l_{6}\right) & \left(s_{4}, l_{8}\right) & \left(s_{4}, l_{4}\right) \\
\left(s_{0}, l_{4}\right) & \left(s_{4}, l_{4}\right) & \left(s_{4}, l_{8}\right)
\end{array}\right)
\end{aligned}
$$

Based on the first stage model (13), we can obtain the value of objective function $z_{1}=2.87$. Based on the second stage model (14), we can obtain $z_{2}=1.13$ and the collective preference vector $w^{c}=(0.34,0.54,0.12)^{T}$.

\subsection{Example 3}

The third example includes six decision makers $e_{k}(k=1,2, \ldots, 6)$ and five alternatives $x_{i}(i=1,2, \ldots, 5)$. Suppose that $c_{k}=1 / 6, k=1,2, \ldots, 6$. The decision makers $e_{1}$ and $e_{2}$ provide the multiplicative preference relations with self-confidence $A^{* 1}$ and $A^{* 2}$, the decision makers $e_{2}$ and $e_{3}$ provide the additive preference relations with self-confidence $P^{* 3}$ and $P^{* 4}$, the decision makers $e_{5}$ and $e_{6}$ provide the ordinal 2-tuple linguistic preference relations with self-confidence $T^{* 5}$ and $T^{* 6}$. 


$$
\begin{aligned}
& A^{* 1}=\left(\begin{array}{lllll}
\left(1, l_{8}\right) & \left(1, l_{7}\right) & \left(4, l_{5}\right) & \left(5, l_{2}\right) & \left(5, l_{0}\right) \\
\left(1, l_{7}\right) & \left(1, l_{8}\right) & \left(4, l_{2}\right) & \left(7, l_{4}\right) & \left(3, l_{1}\right) \\
\left(\frac{1}{4}, l_{5}\right) & \left(\frac{1}{4}, l_{2}\right) & \left(1, l_{8}\right) & \left(2, l_{2}\right) & \left(2, l_{4}\right) \\
\left(\frac{1}{5}, l_{2}\right) & \left(\frac{1}{7}, l_{4}\right) & \left(\frac{1}{2}, l_{2}\right) & \left(1, l_{8}\right) & \left(2, l_{3}\right) \\
\left(\frac{1}{5}, l_{0}\right) & \left(\frac{1}{3}, l_{1}\right) & \left(\frac{1}{2}, l_{4}\right) & \left(\frac{1}{2}, l_{3}\right) & \left(1, l_{8}\right)
\end{array}\right) \\
& A^{* 2}=\left(\begin{array}{lllll}
\left(1, l_{8}\right) & \left(1, l_{1}\right) & \left(2, l_{4}\right) & \left(3, l_{2}\right) & \left(4, l_{2}\right) \\
\left(1, l_{1}\right) & \left(1, l_{8}\right) & \left(3, l_{3}\right) & \left(3, l_{5}\right) & \left(3, l_{1}\right) \\
\left(\frac{1}{2}, l_{4}\right) & \left(\frac{1}{3}, l_{3}\right) & \left(1, l_{8}\right) & \left(2, l_{2}\right) & \left(2, l_{4}\right) \\
\left(\frac{1}{3}, l_{2}\right) & \left(\frac{1}{3}, l_{5}\right) & \left(\frac{1}{2}, l_{2}\right) & \left(1, l_{8}\right) & \left(2, l_{1}\right) \\
\left(\frac{1}{4}, l_{2}\right) & \left(\frac{1}{3}, l_{1}\right) & \left(\frac{1}{2}, l_{4}\right) & \left(\frac{1}{2}, l_{1}\right) & \left(1, l_{8}\right)
\end{array}\right) \\
& P^{* 3}=\left(\begin{array}{ccccc}
\left(0.5, l_{8}\right) & \left(0.53, l_{3}\right) & \left(0.56, l_{3}\right) & \left(0.56, l_{2}\right) & \left(0.6, l_{1}\right) \\
\left(0.47, l_{3}\right) & \left(0.5, l_{8}\right) & \left(0.8, l_{5}\right) & \left(0.6, l_{1}\right) & \left(0.8, l_{4}\right) \\
\left(0.44, l_{3}\right) & \left(0.2, l_{5}\right) & \left(0.5, l_{8}\right) & \left(0.6, l_{3}\right) & \left(0.7, l_{7}\right) \\
\left(0.44, l_{2}\right) & \left(0.4, l_{1}\right) & \left(0.4, l_{3}\right) & \left(0.5, l_{8}\right) & \left(0.6, l_{0}\right) \\
\left(0.4, l_{1}\right) & \left(0.2, l_{4}\right) & \left(0.3, l_{7}\right) & \left(0.4, l_{0}\right) & \left(0.5, l_{8}\right)
\end{array}\right) \\
& P^{* 4}=\left(\begin{array}{ccccc}
\left(0.5, l_{8}\right) & \left(0.7, l_{4}\right) & \left(0.75, l_{3}\right) & \left(0.95, l_{2}\right) & \left(0.6, l_{3}\right) \\
\left(0.3, l_{4}\right) & \left(0.5, l_{8}\right) & \left(0.55, l_{2}\right) & \left(0.8, l_{1}\right) & \left(0.5, l_{5}\right) \\
\left(0.25, l_{3}\right) & \left(0.45, l_{2}\right) & \left(0.5, l_{8}\right) & \left(0.7, l_{5}\right) & \left(0.6, l_{5}\right) \\
\left(0.05, l_{2}\right) & \left(0.2, l_{1}\right) & \left(0.3, l_{5}\right) & \left(0.5, l_{8}\right) & \left(0.85, l_{3}\right) \\
\left(0.4, l_{3}\right) & \left(0.5, l_{5}\right) & \left(0.4, l_{5}\right) & \left(0.15, l_{3}\right) & \left(0.5, l_{8}\right)
\end{array}\right) \\
& T^{* 5}=\left(\begin{array}{lllll}
\left(s_{4}, l_{8}\right) & \left(s_{6}, l_{6}\right) & \left(s_{8}, l_{1}\right) & \left(s_{7}, l_{5}\right) & \left(s_{5}, l_{8}\right) \\
\left(s_{2}, l_{6}\right) & \left(s_{4}, l_{8}\right) & \left(s_{6}, l_{2}\right) & \left(s_{7}, l_{3}\right) & \left(s_{7}, l_{4}\right) \\
\left(s_{0}, l_{1}\right) & \left(s_{2}, l_{2}\right) & \left(s_{4}, l_{8}\right) & \left(s_{8}, l_{7}\right) & \left(s_{7}, l_{2}\right) \\
\left(s_{1}, l_{5}\right) & \left(s_{1}, l_{3}\right) & \left(s_{0}, l_{7}\right) & \left(s_{4}, l_{8}\right) & \left(s_{6}, l_{1}\right) \\
\left(s_{3}, l_{8}\right) & \left(s_{1}, l_{4}\right) & \left(s_{1}, l_{2}\right) & \left(s_{2}, l_{1}\right) & \left(s_{4}, l_{8}\right)
\end{array}\right) \\
& T^{* 6}=\left(\begin{array}{lllll}
\left(s_{4}, l_{8}\right) & \left(s_{7}, l_{2}\right) & \left(s_{7}, l_{4}\right) & \left(s_{5}, l_{5}\right) & \left(s_{6}, l_{3}\right) \\
\left(s_{1}, l_{2}\right) & \left(s_{4}, l_{8}\right) & \left(s_{5}, l_{7}\right) & \left(s_{7}, l_{6}\right) & \left(s_{8}, l_{1}\right) \\
\left(s_{1}, l_{4}\right) & \left(s_{3}, l_{7}\right) & \left(s_{4}, l_{8}\right) & \left(s_{5}, l_{3}\right) & \left(s_{5}, l_{3}\right) \\
\left(s_{3}, l_{5}\right) & \left(s_{1}, l_{6}\right) & \left(s_{3}, l_{3}\right) & \left(s_{4}, l_{8}\right) & \left(s_{6}, l_{2}\right) \\
\left(s_{2}, l_{3}\right) & \left(s_{0}, l_{1}\right) & \left(s_{3}, l_{3}\right) & \left(s_{2}, l_{2}\right) & \left(s_{4}, l_{8}\right)
\end{array}\right)
\end{aligned}
$$


Based on the first stage model (13), we can obtain the value of objective function $z_{1}=3.43$. Based on the second stage model (14), we can obtain $z_{2}=0.50$ and the collective preference vector $w^{c}=(0.40,0.34,0.20,0.05,0.01)^{T}$.

\subsection{Comparative analysis}

In this subsection, we study the influence of different self-confidence levels on the GDM results. Consider the following six matrices $A_{1}^{* 1}, P_{1}^{*_{2}}, T_{1}^{* 3}, A_{2}^{* 1}, P_{2}^{*_{2}}$ and $T_{2}^{* 3}$. The matrices $A_{1}^{* 1}$ and $A_{2}^{*_{1}}$ have the same preference values but different self-confidence levels with matrix $A^{*_{1}}$ in Example 1. The matrices $P_{1}^{*_{2}}$ and $P_{2}^{*_{2}}$ have the same preference values but different self-confidence levels with matrix $P^{* 2}$ in Example 1. The matrices $T_{1}^{* 3}$ and $T_{2}^{* 3}$ have the same preference values but different self-confidence levels with matrix $T^{* 3}$ in Example 1. The corresponding matrices are given as follows,

$$
\begin{aligned}
& A_{1}^{* 1}=\left(\begin{array}{cccc}
\left(1, l_{8}\right) & \left(2, l_{4}\right) & \left(\frac{1}{3}, l_{6}\right) & \left(4, l_{7}\right) \\
\left(\frac{1}{2}, l_{4}\right) & \left(1, l_{8}\right) & \left(\frac{1}{3}, l_{2}\right) & \left(\frac{1}{4}, l_{3}\right) \\
\left(3, l_{6}\right) & \left(3, l_{2}\right) & \left(1, l_{8}\right) & \left(7, l_{0}\right) \\
\left(\frac{1}{4}, l_{7}\right) & \left(4, l_{3}\right) & \left(\frac{1}{7}, l_{0}\right) & \left(1, l_{8}\right)
\end{array}\right) \quad A_{2}^{* 1}=\left(\begin{array}{cccc}
\left(1, l_{8}\right) & \left(2, l_{8}\right) & \left(\frac{1}{3}, l_{8}\right) & \left(4, l_{8}\right) \\
\left(\frac{1}{2}, l_{8}\right) & \left(1, l_{8}\right) & \left(\frac{1}{3}, l_{8}\right) & \left(\frac{1}{4}, l_{8}\right) \\
\left(3, l_{8}\right) & \left(3, l_{8}\right) & \left(1, l_{8}\right) & \left(7, l_{8}\right) \\
\left(\frac{1}{4}, l_{8}\right) & \left(4, l_{8}\right) & \left(\frac{1}{7}, l_{8}\right) & \left(1, l_{8}\right)
\end{array}\right) \\
& P_{1}^{* 2}=\left(\begin{array}{llll}
\left(0.5, l_{8}\right) & \left(0.5, l_{4}\right) & \left(0.6, l_{2}\right) & \left(0.9, l_{5}\right) \\
\left(0.5, l_{4}\right) & \left(0.5, l_{8}\right) & \left(0.8, l_{6}\right) & \left(0.6, l_{6}\right) \\
\left(0.4, l_{2}\right) & \left(0.2, l_{6}\right) & \left(0.5, l_{8}\right) & \left(0.8, l_{3}\right) \\
\left(0.1, l_{5}\right) & \left(0.4, l_{6}\right) & \left(0.2, l_{3}\right) & \left(0.5, l_{8}\right)
\end{array}\right) \quad P_{2}^{* 2}=\left(\begin{array}{llll}
\left(0.5, l_{8}\right) & \left(0.5, l_{8}\right) & \left(0.6, l_{8}\right) & \left(0.9, l_{8}\right) \\
\left(0.5, l_{8}\right) & \left(0.5, l_{8}\right) & \left(0.8, l_{8}\right) & \left(0.6, l_{8}\right) \\
\left(0.4, l_{8}\right) & \left(0.2, l_{8}\right) & \left(0.5, l_{8}\right) & \left(0.8, l_{8}\right) \\
\left(0.1, l_{8}\right) & \left(0.4, l_{8}\right) & \left(0.2, l_{8}\right) & \left(0.5, l_{8}\right)
\end{array}\right) \\
& T_{1}^{* 3}=\left(\begin{array}{llll}
\left(s_{4}, l_{8}\right) & \left(s_{1}, l_{4}\right) & \left(s_{1}, l_{7}\right) & \left(s_{4}, l_{1}\right) \\
\left(s_{7}, l_{4}\right) & \left(s_{4}, l_{8}\right) & \left(s_{6}, l_{3}\right) & \left(s_{4}, l_{2}\right) \\
\left(s_{7}, l_{7}\right) & \left(s_{2}, l_{3}\right) & \left(s_{4}, l_{8}\right) & \left(s_{3}, l_{6}\right) \\
\left(s_{4}, l_{1}\right) & \left(s_{4}, l_{2}\right) & \left(s_{5}, l_{6}\right) & \left(s_{4}, l_{8}\right)
\end{array}\right) \quad T_{2}^{* 3}=\left(\begin{array}{llll}
\left(s_{4}, l_{8}\right) & \left(s_{1}, l_{8}\right) & \left(s_{1}, l_{8}\right) & \left(s_{4}, l_{8}\right) \\
\left(s_{7}, l_{8}\right) & \left(s_{4}, l_{8}\right) & \left(s_{6}, l_{8}\right) & \left(s_{4}, l_{8}\right) \\
\left(s_{7}, l_{8}\right) & \left(s_{2}, l_{8}\right) & \left(s_{4}, l_{8}\right) & \left(s_{3}, l_{8}\right) \\
\left(s_{4}, l_{8}\right) & \left(s_{4}, l_{8}\right) & \left(s_{5}, l_{8}\right) & \left(s_{4}, l_{8}\right)
\end{array}\right)
\end{aligned}
$$

The six matrices contain two groups: the one group is the matrices $A_{1}^{* 1}, P_{1}^{*_{2}}$ and $T_{1}^{* 3}$, the other group is the matrices $A_{2}^{*_{1}}, P_{2}^{*_{2}}$ and $T_{2}^{* 3}$. Using the two-stage linear programming model obtains the GDM results for each group. The value of objective function $z_{1}$, the value 
of objective function $z_{2}$ and the collective preference vectors of three groups for Example 1 are presented in the Table 1.

Table 1 The comparison results regarding the three groups for Example 1

\begin{tabular}{cccc}
\hline & $z_{1}$ & $z_{2}$ & $w^{c}$ \\
\hline$\left(A^{* 1}, P^{* 2}, T^{* 3}\right)$ & 2.72 & 0.71 & $(0.26,0.26,0.42,0.06)^{T}$ \\
$\left(A_{1}^{* 1}, P_{1}^{* 2}, T_{1}^{* 3}\right)$ & 3.40 & 0.84 & $(0.40,0.30,0.20,0.10)^{T}$ \\
$\left(A_{2}^{* 1}, P_{2}^{* 2}, T_{2}^{* 3}\right)$ & 5.87 & 1.15 & $(0.46,0.24,0.26,0.04)^{T}$ \\
\hline
\end{tabular}

Furthermore, we make the equivalent comparative analysis for the GDM of Example 2. Consider the following eight matrices $A_{1}^{* 1}, P_{1}^{* 2}, P_{1}^{* 3}, T_{1}^{* 4}, A_{2}^{* 1}, P_{2}^{* 2}, P_{2}^{* 3}$ and $T_{2}^{* 4}$. The corresponding matrices are given as follows,

$$
\begin{array}{ll}
A_{1}^{* 1}=\left(\begin{array}{lll}
\left(1, l_{8}\right) & \left(2, l_{7}\right) & \left(3, l_{2}\right) \\
\left(\frac{1}{2}, l_{7}\right) & \left(1, l_{8}\right) & \left(\frac{1}{4}, l_{6}\right) \\
\left(\frac{1}{3}, l_{2}\right) & \left(4, l_{6}\right) & \left(1, l_{8}\right)
\end{array}\right) & A_{2}^{* 1}=\left(\begin{array}{ccc}
\left(1, l_{8}\right) & \left(2, l_{8}\right) & \left(3, l_{8}\right) \\
\left(\frac{1}{2}, l_{8}\right) & \left(1, l_{8}\right) & \left(\frac{1}{4}, l_{8}\right) \\
\left(\frac{1}{3}, l_{8}\right) & \left(4, l_{8}\right) & \left(1, l_{8}\right)
\end{array}\right) \\
P_{1}^{* 2}=\left(\begin{array}{lll}
\left(0.5, l_{8}\right) & \left(0.3, l_{2}\right) & \left(0.4, l_{5}\right) \\
\left(0.7, l_{2}\right) & \left(0.5, l_{8}\right) & \left(0.2, l_{4}\right) \\
\left(0.6, l_{5}\right) & \left(0.8, l_{4}\right) & \left(0.5, l_{8}\right)
\end{array}\right) & P_{2}^{* 2}=\left(\begin{array}{lll}
\left(0.5, l_{8}\right) & \left(0.3, l_{8}\right) & \left(0.4, l_{8}\right) \\
\left(0.7, l_{8}\right) & \left(0.5, l_{8}\right) & \left(0.2, l_{8}\right) \\
\left(0.6, l_{8}\right) & \left(0.8, l_{8}\right) & \left(0.5, l_{8}\right)
\end{array}\right) \\
P_{1}^{* 3}=\left(\begin{array}{lll}
\left(0.5, l_{8}\right) & \left(0.2, l_{3}\right) & \left(0.6, l_{3}\right) \\
\left(0.8, l_{3}\right) & \left(0.5, l_{8}\right) & \left(0.7, l_{6}\right) \\
\left(0.4, l_{3}\right) & \left(0.3, l_{6}\right) & \left(0.5, l_{8}\right)
\end{array}\right) & P_{2}^{* 3}=\left(\begin{array}{lll}
\left(0.5, l_{8}\right) & \left(0.2, l_{8}\right) & \left(0.6, l_{8}\right) \\
\left(0.8, l_{8}\right) & \left(0.5, l_{8}\right) & \left(0.7, l_{8}\right) \\
\left(0.4, l_{8}\right) & \left(0.3, l_{8}\right) & \left(0.5, l_{8}\right)
\end{array}\right) \\
T_{1}^{* 4}=\left(\begin{array}{lll}
\left(s_{4}, l_{8}\right) & \left(s_{3}, l_{5}\right) & \left(s_{8}, l_{1}\right) \\
\left(s_{5}, l_{5}\right) & \left(s_{4}, l_{8}\right) & \left(s_{4}, l_{8}\right) \\
\left(s_{0}, l_{1}\right) & \left(s_{4}, l_{8}\right) & \left(s_{4}, l_{8}\right)
\end{array}\right) & T_{2}^{* 4}=\left(\begin{array}{lll}
\left(s_{4}, l_{8}\right) & \left(s_{3}, l_{8}\right) & \left(s_{8}, l_{8}\right) \\
\left(s_{5}, l_{8}\right) & \left(s_{4}, l_{8}\right) & \left(s_{4}, l_{8}\right) \\
\left(s_{0}, l_{8}\right) & \left(s_{4}, l_{8}\right) & \left(s_{4}, l_{8}\right)
\end{array}\right)
\end{array}
$$

The eight matrices contain two groups: the one group is the matrices $A_{1}^{* 1}, P_{1}^{* 2}, P_{1}^{* 3}$ and $T_{1}^{* 4}$, the other group is the matrices $A_{2}^{* 1}, P_{2}^{* 2}, P_{2}^{* 3}$ and $T_{2}^{* 4}$. The comparison results regarding three groups for Example 2 are presented in the Table 2. 
Table 2 The comparison results regarding the three groups for Example 2

\begin{tabular}{cccc}
\hline & $z_{1}$ & $z_{2}$ & $w^{c}$ \\
\hline$\left(A^{* 1}, P^{* 2}, P^{* 3}, T^{* 4}\right)$ & 2.87 & 1.13 & $(0.34,0.54,0.12)^{T}$ \\
$\left(A_{1}^{* 1}, P_{1}^{* 2}, P_{1}^{* 3}, T_{1}^{* 4}\right)$ & 1.89 & 0.53 & $(0.32,0.16,0.52)^{T}$ \\
$\left(A_{2}^{* 1}, P_{2}^{* 2}, P_{2}^{* 3}, T_{2}^{* 4}\right)$ & 5.06 & 2.03 & $(0.20,0.40,0.40)^{T}$ \\
\hline
\end{tabular}

Finally, we make the equivalent comparative analysis for the GDM of Example 3. Consider the following twelve matrices which have the same preference values but different self-confidence levels with the corresponding matrices of Example 3.

$$
\begin{aligned}
& A_{1}^{* 1}=\left(\begin{array}{lllll}
\left(1, l_{8}\right) & \left(1, l_{8}\right) & \left(4, l_{5}\right) & \left(5, l_{6}\right) & \left(5, l_{7}\right) \\
\left(1, l_{8}\right) & \left(1, l_{8}\right) & \left(4, l_{5}\right) & \left(7, l_{4}\right) & \left(3, l_{6}\right) \\
\left(\frac{1}{4}, l_{5}\right) & \left(\frac{1}{4}, l_{5}\right) & \left(1, l_{8}\right) & \left(2, l_{7}\right) & \left(2, l_{2}\right) \\
\left(\frac{1}{5}, l_{6}\right) & \left(\frac{1}{7}, l_{4}\right) & \left(\frac{1}{2}, l_{7}\right) & \left(1, l_{8}\right) & \left(2, l_{1}\right) \\
\left(\frac{1}{5}, l_{7}\right) & \left(\frac{1}{3}, l_{6}\right) & \left(\frac{1}{2}, l_{2}\right) & \left(\frac{1}{2}, l_{1}\right) & \left(1, l_{8}\right)
\end{array}\right) \\
& A_{2}^{* 1}=\left(\begin{array}{lllll}
\left(1, l_{8}\right) & \left(1, l_{8}\right) & \left(4, l_{8}\right) & \left(5, l_{8}\right) & \left(5, l_{8}\right) \\
\left(1, l_{8}\right) & \left(1, l_{8}\right) & \left(4, l_{8}\right) & \left(7, l_{8}\right) & \left(3, l_{8}\right) \\
\left(\frac{1}{4}, l_{8}\right) & \left(\frac{1}{4}, l_{8}\right) & \left(1, l_{8}\right) & \left(2, l_{8}\right) & \left(2, l_{8}\right) \\
\left(\frac{1}{5}, l_{8}\right) & \left(\frac{1}{7}, l_{8}\right) & \left(\frac{1}{2}, l_{8}\right) & \left(1, l_{8}\right) & \left(2, l_{8}\right) \\
\left(\frac{1}{5}, l_{8}\right) & \left(\frac{1}{3}, l_{8}\right) & \left(\frac{1}{2}, l_{8}\right) & \left(\frac{1}{2}, l_{8}\right) & \left(1, l_{8}\right)
\end{array}\right) \\
& \left(\begin{array}{llllll}
\left(1, l_{8}\right) & \left(1, l_{7}\right) & \left(2, l_{7}\right) & \left(3, l_{4}\right) & \left(4, l_{6}\right) \\
\left(1, l_{7}\right) & \left(1, l_{8}\right) & \left(3, l_{5}\right) & \left(3, l_{8}\right) & \left(3, l_{6}\right) \\
\left(\frac{1}{2}, l_{7}\right) & \left(\frac{1}{3}, l_{5}\right) & \left(1, l_{8}\right) & \left(2, l_{7}\right) & \left(2, l_{5}\right) \\
\left(\frac{1}{3}, l_{4}\right) & \left(\frac{1}{3}, l_{8}\right) & \left(\frac{1}{2}, l_{7}\right) & \left(1, l_{8}\right) & \left(2, l_{8}\right) \\
\left(\frac{1}{4}, l_{6}\right) & \left(\frac{1}{3}, l_{6}\right) & \left(\frac{1}{2}, l_{5}\right) & \left(\frac{1}{2}, l_{8}\right) & \left(1, l_{8}\right)
\end{array}\right)
\end{aligned}
$$




$$
\begin{aligned}
& A_{2}^{* 2}=\left(\begin{array}{ccccc}
\left(1, l_{8}\right) & \left(1, l_{8}\right) & \left(2, l_{8}\right) & \left(3, l_{8}\right) & \left(4, l_{8}\right) \\
\left(1, l_{8}\right) & \left(1, l_{8}\right) & \left(3, l_{8}\right) & \left(3, l_{8}\right) & \left(3, l_{8}\right) \\
\left(\frac{1}{2}, l_{8}\right) & \left(\frac{1}{3}, l_{8}\right) & \left(1, l_{8}\right) & \left(2, l_{8}\right) & \left(2, l_{8}\right) \\
\left(\frac{1}{3}, l_{8}\right) & \left(\frac{1}{3}, l_{8}\right) & \left(\frac{1}{2}, l_{8}\right) & \left(1, l_{8}\right) & \left(2, l_{8}\right) \\
\left(\frac{1}{4}, l_{8}\right) & \left(\frac{1}{3}, l_{8}\right) & \left(\frac{1}{2}, l_{8}\right) & \left(\frac{1}{2}, l_{8}\right) & \left(1, l_{8}\right)
\end{array}\right) \\
& P_{1}^{* 3}=\left(\begin{array}{ccccc}
\left(0.5, l_{8}\right) & \left(0.53, l_{7}\right) & \left(0.56, l_{8}\right) & \left(0.56, l_{6}\right) & \left(0.6, l_{8}\right) \\
\left(0.47, l_{7}\right) & \left(0.5, l_{8}\right) & \left(0.8, l_{5}\right) & \left(0.6, l_{5}\right) & \left(0.8, l_{8}\right) \\
\left(0.44, l_{8}\right) & \left(0.2, l_{5}\right) & \left(0.5, l_{8}\right) & \left(0.6, l_{6}\right) & \left(0.7, l_{1}\right) \\
\left(0.44, l_{6}\right) & \left(0.4, l_{5}\right) & \left(0.4, l_{6}\right) & \left(0.5, l_{8}\right) & \left(0.6, l_{6}\right) \\
\left(0.4, l_{8}\right) & \left(0.2, l_{8}\right) & \left(0.3, l_{1}\right) & \left(0.4, l_{6}\right) & \left(0.5, l_{8}\right)
\end{array}\right) \\
& P_{2}^{* 3}=\left(\begin{array}{ccccc}
\left(0.5, l_{8}\right) & \left(0.53, l_{8}\right) & \left(0.56, l_{8}\right) & \left(0.56, l_{8}\right) & \left(0.6, l_{8}\right) \\
\left(0.47, l_{8}\right) & \left(0.5, l_{8}\right) & \left(0.8, l_{8}\right) & \left(0.6, l_{8}\right) & \left(0.8, l_{8}\right) \\
\left(0.44, l_{8}\right) & \left(0.2, l_{8}\right) & \left(0.5, l_{8}\right) & \left(0.6, l_{8}\right) & \left(0.7, l_{8}\right) \\
\left(0.44, l_{8}\right) & \left(0.4, l_{8}\right) & \left(0.4, l_{8}\right) & \left(0.5, l_{8}\right) & \left(0.6, l_{8}\right) \\
\left(0.4, l_{8}\right) & \left(0.2, l_{8}\right) & \left(0.3, l_{8}\right) & \left(0.4, l_{8}\right) & \left(0.5, l_{8}\right)
\end{array}\right) \\
& P_{1}^{* 4}=\left(\begin{array}{ccccc}
\left(0.5, l_{8}\right) & \left(0.7, l_{4}\right) & \left(0.75, l_{5}\right) & \left(0.95, l_{5}\right) & \left(0.6, l_{6}\right) \\
\left(0.3, l_{4}\right) & \left(0.5, l_{8}\right) & \left(0.55, l_{8}\right) & \left(0.8, l_{7}\right) & \left(0.5, l_{7}\right) \\
\left(0.25, l_{5}\right) & \left(0.45, l_{8}\right) & \left(0.5, l_{8}\right) & \left(0.7, l_{7}\right) & \left(0.6, l_{6}\right) \\
\left(0.05, l_{5}\right) & \left(0.2, l_{7}\right) & \left(0.3, l_{7}\right) & \left(0.5, l_{8}\right) & \left(0.85, l_{5}\right) \\
\left(0.4, l_{6}\right) & \left(0.5, l_{7}\right) & \left(0.4, l_{6}\right) & \left(0.15, l_{5}\right) & \left(0.5, l_{8}\right)
\end{array}\right) \\
& P_{2}^{* 4}=\left(\begin{array}{ccccc}
\left(0.5, l_{8}\right) & \left(0.7, l_{8}\right) & \left(0.75, l_{8}\right) & \left(0.95, l_{8}\right) & \left(0.6, l_{8}\right) \\
\left(0.3, l_{8}\right) & \left(0.5, l_{8}\right) & \left(0.55, l_{8}\right) & \left(0.8, l_{8}\right) & \left(0.5, l_{8}\right) \\
\left(0.25, l_{8}\right) & \left(0.45, l_{8}\right) & \left(0.5, l_{8}\right) & \left(0.7, l_{8}\right) & \left(0.6, l_{8}\right) \\
\left(0.05, l_{8}\right) & \left(0.2, l_{8}\right) & \left(0.3, l_{8}\right) & \left(0.5, l_{8}\right) & \left(0.85, l_{8}\right) \\
\left(0.4, l_{8}\right) & \left(0.5, l_{8}\right) & \left(0.4, l_{8}\right) & \left(0.15, l_{8}\right) & \left(0.5, l_{8}\right)
\end{array}\right) \\
& T_{1}^{* 5}=\left(\begin{array}{lllll}
\left(s_{4}, l_{8}\right) & \left(s_{6}, l_{6}\right) & \left(s_{8}, l_{8}\right) & \left(s_{7}, l_{7}\right) & \left(s_{5}, l_{6}\right) \\
\left(s_{2}, l_{6}\right) & \left(s_{4}, l_{8}\right) & \left(s_{6}, l_{5}\right) & \left(s_{7}, l_{5}\right) & \left(s_{7}, l_{8}\right) \\
\left(s_{0}, l_{8}\right) & \left(s_{2}, l_{5}\right) & \left(s_{4}, l_{8}\right) & \left(s_{8}, l_{3}\right) & \left(s_{7}, l_{5}\right) \\
\left(s_{1}, l_{7}\right) & \left(s_{1}, l_{5}\right) & \left(s_{0}, l_{3}\right) & \left(s_{4}, l_{8}\right) & \left(s_{6}, l_{6}\right) \\
\left(s_{3}, l_{6}\right) & \left(s_{1}, l_{8}\right) & \left(s_{1}, l_{5}\right) & \left(s_{2}, l_{6}\right) & \left(s_{4}, l_{8}\right)
\end{array}\right) \\
& T_{2}^{* 5}=\left(\begin{array}{lllll}
\left(s_{4}, l_{8}\right) & \left(s_{6}, l_{8}\right) & \left(s_{8}, l_{8}\right) & \left(s_{7}, l_{8}\right) & \left(s_{5}, l_{8}\right) \\
\left(s_{2}, l_{8}\right) & \left(s_{4}, l_{8}\right) & \left(s_{6}, l_{8}\right) & \left(s_{7}, l_{8}\right) & \left(s_{7}, l_{8}\right) \\
\left(s_{0}, l_{8}\right) & \left(s_{2}, l_{8}\right) & \left(s_{4}, l_{8}\right) & \left(s_{8}, l_{8}\right) & \left(s_{7}, l_{8}\right) \\
\left(s_{1}, l_{8}\right) & \left(s_{1}, l_{8}\right) & \left(s_{0}, l_{8}\right) & \left(s_{4}, l_{8}\right) & \left(s_{6}, l_{8}\right) \\
\left(s_{3}, l_{8}\right) & \left(s_{1}, l_{8}\right) & \left(s_{1}, l_{8}\right) & \left(s_{2}, l_{8}\right) & \left(s_{4}, l_{8}\right)
\end{array}\right)
\end{aligned}
$$




$$
\begin{gathered}
T_{1}^{* 6}=\left(\begin{array}{lllll}
\left(s_{4}, l_{8}\right) & \left(s_{7}, l_{7}\right) & \left(s_{7}, l_{7}\right) & \left(s_{5}, l_{6}\right) & \left(s_{6}, l_{5}\right) \\
\left(s_{1}, l_{7}\right) & \left(s_{4}, l_{8}\right) & \left(s_{5}, l_{8}\right) & \left(s_{7}, l_{4}\right) & \left(s_{8}, l_{3}\right) \\
\left(s_{1}, l_{7}\right) & \left(s_{3}, l_{8}\right) & \left(s_{4}, l_{8}\right) & \left(s_{5}, l_{5}\right) & \left(s_{5}, l_{8}\right) \\
\left(s_{3}, l_{6}\right) & \left(s_{1}, l_{4}\right) & \left(s_{3}, l_{5}\right) & \left(s_{4}, l_{8}\right) & \left(s_{6}, l_{7}\right) \\
\left(s_{2}, l_{5}\right) & \left(s_{0}, l_{3}\right) & \left(s_{3}, l_{8}\right) & \left(s_{2}, l_{7}\right) & \left(s_{4}, l_{8}\right)
\end{array}\right) \\
T_{2}^{* 6}=\left(\begin{array}{lllll}
\left(s_{4}, l_{8}\right) & \left(s_{7}, l_{8}\right) & \left(s_{7}, l_{8}\right) & \left(s_{5}, l_{8}\right) & \left(s_{6}, l_{8}\right) \\
\left(s_{1}, l_{8}\right) & \left(s_{4}, l_{8}\right) & \left(s_{5}, l_{8}\right) & \left(s_{7}, l_{8}\right) & \left(s_{8}, l_{8}\right) \\
\left(s_{1}, l_{8}\right) & \left(s_{3}, l_{8}\right) & \left(s_{4}, l_{8}\right) & \left(s_{5}, l_{8}\right) & \left(s_{5}, l_{8}\right) \\
\left(s_{3}, l_{8}\right) & \left(s_{1}, l_{8}\right) & \left(s_{3}, l_{8}\right) & \left(s_{4}, l_{8}\right) & \left(s_{6}, l_{8}\right) \\
\left(s_{2}, l_{8}\right) & \left(s_{0}, l_{8}\right) & \left(s_{3}, l_{8}\right) & \left(s_{2}, l_{8}\right) & \left(s_{4}, l_{8}\right)
\end{array}\right)
\end{gathered}
$$

The twelve matrices also contain two groups: the one group is the matrices $A_{1}^{* 1}, A_{1}^{* 2}$, $P_{1}^{* 3}, P_{1}^{* 4}, T_{1}^{* 5}$ and $T_{1}^{* 6}$, the other group is the matrices $A_{2}^{* 1}, A_{2}^{* 2}, P_{2}^{* 3}, P_{2}^{* 4}, T_{2}^{* 5}$ and $T_{2}^{* 6}$. The comparison results regarding the three groups for Example 3 are presented in the Table 3.

Table 3 The comparison results regarding the three groups for Example 3

\begin{tabular}{cccc}
\hline & $z_{1}$ & $z_{2}$ & $w^{c}$ \\
\hline$\left(A^{*_{1}}, A^{*_{2}}, P^{*_{3}}, P^{* 4}, T^{* 5}, T^{* 6}\right)$ & 3.43 & 0.50 & $(0.40,0.34,0.20,0.05,0.01)^{T}$ \\
$\left(A_{1}^{* 1}, A_{1}^{* 2}, P_{1}^{* 3}, P_{1}^{* 4}, T_{1}^{* 5}, T_{1}^{* 6}\right)$ & 5.70 & 0.64 & $(0.32,0.30,0.20,0.10,0.08)^{T}$ \\
$\left(A_{2}^{* 1}, A_{2}^{* 2}, P_{2}^{* 3}, P_{2}^{* 4}, T_{2}^{* 5}, T_{2}^{* 6}\right)$ & 8.08 & 0.82 & $(0.36,0.30,0.18,0.09,0.07)^{T}$ \\
\hline
\end{tabular}

From Table 1, Table 2 and Table 3, we notice that different self-confidence levels lead to different collective preference vectors. Thus the self-confidence levels have certain influence on the GDM results.

\section{Conclusions}

In this study, we define a new kind of preference relation, called preference relation with self-confidence. Then, we present a two-stage linear programming model to deal with the GDM problem based on heterogeneous preference relations with self-confidence. The main contributions presented are as follows:

(1) This study defines the preference relations with self-confidence, which allow decision makers to have multiple self-confidence levels to express their preferences regarding pairs of alternatives. 
(2) We propose a two-stage linear programming model for estimating the collective preference vector in the GDM based on heterogeneous preference relations with self-confidence.

Finally, a comparison study is conducted to demonstrate the influence of self-confidence levels on the GDM results. It will be an interesting future research to find out possible relationships among preferences, self-confidence assessments and results. Meanwhile, the consensus problem is a hot topic in GDM [2, 7, 10, 17, 32, 37], and it will be interesting to investigate the consensus reaching model in GDM based on heterogeneous preference relations with self-confidence.

\section{Acknowledgments}

This work was supported in part by NSF of China under Grants Nos. 71171160 and 71571124, FEDER funds under Grant TIN2013-40658-P, and the Andalusian Excellence Project Grant TIC-5991.

\section{References}

[1] S. Alonso, F. Chiclana, F. Herrera, E. Herrera-Viedma, J. Alcalá-Fdez, C. Porcel. A consistency-based procedure to estimate missing pairwise preference values. International Journal of Intelligent Systems 23 (2008) 155-175.

[2] S. Alonso, I. J. Pérez, F. J. Cabrerizo, E. Herrera-Viedma. A Linguistic Consensus Model for Web 2.0 Communities. Applied Soft Computing 13 (1) (2013) 149-157.

[3] X. Chen, H. J. Zhang, Y. C. Dong. The fusion process with heterogeneous preference structures in group decision making: A survey. Information Fusion 24 (2015) 72-83.

[4] F. Chiclana, F. Herrera, E. Herrera-Viedma. Integrating three representation models in fuzzy multipurpose decision making based on fuzzy preference relations. Fuzzy Sets and Systems 97 (1998) 33-48.

[5] F. Chiclana, F. Herrera, E. Herrera-Viedma. Integrating multiplicative preference relations in a multipurpose decision-making model based on fuzzy preference relations. Fuzzy Sets and Systems 122 (2001) 277-291.

[6] F. Chiclana, E. Herrera-Viedma, S. Alonso, F. Herrera. Cardinal consistency of reciprocal preference relations: A Characterization of multiplicative transitivity. IEEE Transactions on Fuzzy Systems 17 (1) (2009) 14-23. 
[7] F. Chiclana, J. M. Tapia Garcia, M. J. Del Moral, E. Herrera-Viedma. A Statistical Comparative Study of Different Similarity Measures of Consensus in Group Decision Making. Information Sciences 221 (2013) 110-123.

[8] F. J. Cabrerizo, S. Alonso, E. Herrera-Viedma. A consensus model for group decision making problems with unbalanced fuzzy linguistic information. International Journal of Information Technology \& Decision making 8 (2009) 109-131.

[9] F. J. Cabrerizo, I. J. Pérez, E. Herrera-Viedma. Managing the consensus in group decision making in an unbalanced fuzzy linguistic context with incomplete information. Knowledge-Based Systems 23 (2010) 169-181.

[10] F. J. Cabrerizo, F. Chiclana, R. Al-Hmouz, A. Morfeq, A. S. Balamash, E. Herrera-Viedma. Fuzzy decision making and consensus: Challenges. Journal of Intelligent \& Fuzzy Systems 29 (3) (2015) 1109-1118.

[11] Y. C. Dong, Y. F. Xu, H. Y. Li. On consistency measures of linguistic preference relations. European Journal of Operational Research 189 (2008) 430-444.

[12] Y. C. Dong, Y. F. Xu, S. Yu. Linguistic multiperson decision making based on the use of multiple preference relations. Fuzzy Sets and Systems 160 (2009) 603-623.

[13] Y. C. Dong, G. Q. Zhang, W. C. Hong, S. Yu. Linguistic computational model based on 2-tuples and intervals. IEEE Transactions on Fuzzy Systems 21 (6) (2013) 1006-1018.

[14] Y. C. Dong, H. J. Zhang. Multiperson decision making with different preference representation structures: A direct consensus framework and its properties. Knowledge-based Systems 58 (2014) 45-57.

[15] Y. C. Dong, E. Herrera-Viedma. Consistency-driven automatic methodology to set interval numerical scales of 2-tuple linguistic term sets and its use in the linguistic GDM with preference relations. IEEE Transactions on Cybernetics 45 (2015) 780-792.

[16] Y. C. Dong, C. C. Li, Y. F. Xu, X. Gu. Consensus-based group decision making under multi-granular unbalanced 2-tuple linguistic preference relations. Group Decision and Negotiation 24 (2015) 217-242.

[17] Y. C. Dong, H. J. Zhang, E. Herrera-Viedma. Integrating experts' weights generated dynamically into the consensus reaching process and its applications in managing non-cooperative behaviors. Decision Support Systems 84 (2016) 1-15. 
[18] Z. P. Fan, S. H. Xiao, G. F. Hu. An optimization method for integrating two kinds of preference information in group decision-making. Computers \& Industrial Engineering 46 (2004) 329-335.

[19] J. Fodor, Roubens. Fuzzy preference modelling and multicriteria decision support. Dordrecht: Kluwer, 1994.

[20] J. L. García-Lapresta, L. C. Meneses. Modeling rationality in a linguistic framework. Fuzzy Sets and Systems 160 (2009) 3211-3223.

[21] E. Herrera-Viedma, F. Herrera, F. Chiclana. A consensus model for multiperson decision making with different preference structures. IEEE Transactions on Systems, Man, and Cybernetics - Part A: Systems and Humans 32 (3) (2002) 394-402.

[22] E. Herrera-Viedma, F. Herrera, F. Chiclana, M. Luque. Some issues on consistency of fuzzy preference relations. European Journal of Operational Research 154 (2004) 98-109.

[23] E. Herrera-Viedma, S. Alonso, F. Chiclana, F. Herrera. A consensus model for group decision making with incomplete fuzzy preference relations. IEEE Transactions on Fuzzy Systems 15 (2007a) 863-877.

[24] E. Herrera-Viedma, F. Chiclana, F. Herrera, S. Alonso. Group decision-making model with incomplete fuzzy preference relations based on additive consistency. IEEE Transactions on Systems, Man, and Cybernetics - Part B, 37 (1) (2007) 176-189.

[25] F. Herrera, L. Martínez. A 2-tuple fuzzy linguistic representation model for computing with words. IEEE Transactions on Fuzzy Systems 8 (2000) 746-752.

[26] F. Herrera, E. Herrera-Viedma, F. Chiclana. Multiperson decision-making based on multiplicative preference relations. European Journal of Operational Research 129 (2001) 372-385.

[27] F. Herrera, S. Alonso, F. Chiclana, and E. Herrera-Viedma. Computing with words in decision making: Foundations, trends and prospects. Fuzzy Optimization and Decision Making, 8 (4) (2009) 337-364.

[28] Y. P. Jiang, Z. P. Fan, J. Ma. A method for group decision making with multigranularity linguistic assessment information. Information Sciences, 178 (4) (2008) 1098-1109.

[29] J. Ma, Z. P. Fan, Y. P. Jiang, J. Y. Mao. An optimization approach to multiperson decision making based on different formats of preference information. IEEE Transactions on Systems, Man and Cybernetics Part A: Systems and Humans 36 (5) (2006) 876-889. 
[30] H. Nurmi. Approaches to collective decision making with fuzzy preference relations. Fuzzy Sets and Systems 6 (1981) 249-259.

[31] S. A. Orlovsky. Decision-making with a fuzzy preference relation. Fuzzy Sets and Systems 1 (3) (1978) 155-167.

[32] I. J. Pérez, F. J. Cabrerizo, E. Herrera-Viedma. A Mobile Decision Support System for Dynamic Group Decision Making Problems. IEEE Transactions on Systems, Man and Cybernetics - Part A: Systems and Humans 40 (6) (2010) 1244-1256.

[33] T. L. Saaty. The Analytical Hierarchy Process. McGraw-Hill, New York, 1980.

[34] T. Tanino. Fuzzy preference orderings in group decision making. Fuzzy Sets and Systems 12 (2) (1984) 117-131.

[35] T. Tanino. Fuzzy preference relations in group decision making, in: Non-Conventional Preference Relations in Decision Making, Springer, Berlin Heidelberg, 1988, pp. 54-71.

[36] T. Tanino. On group decision making under fuzzy preferences, in: Multiperson Decision Making Models using Fuzzy Sets and Possibility Theory, Springer, Netherlands, 1990, pp. $172-185$.

[37] J. M. Tapia García, M. J. Del Moral, M. A. Martínez, E. Herrera-Viedma. A consensus model for group decision making problems with linguistic interval fuzzy preference relations. Expert Systems with Applications 39 (11) (2012) 10022-10030.

[38] R. Ureña, F. Chiclana, J. A. Morente, E. Herrera-Viedma. Managing incomplete preference relations in decision making: A review and future trends. Information Sciences 302 (2015) 14-32.

[39] R. Ureña, F. Chiclana, H. Fujita, E. Herrera-Viedma. Confidence-consistency driven group decision making approach with incomplete reciprocal intuitionistic preference relations. Original Research Article Knowledge-Based Systems 89 (2015) 86-96.

[40] J. Wu, F. Chiclana, E. Herrera-Viedma. Trust based consensus model for social network in an incomplete linguistic information context. Applied Soft Computing 23 (2015) 827-839.

[41] Z. S. Xu. Nonlinear programming model integrating different preference structures. IEEE Transactions on Systems, Man, and Cybernetics - Part A: Systems and Humans 41 (1) (2011) 169-177. 
[42] L.A. Zadeh. A Note on Z-numbers. Information Sciences 181 (2011) 2923-2932. 